\title{
Correction to: Can the application of farm dairy effluent enhance cadmium leachingfrom soil?
}

\section{Colin William Gray ${ }^{1}$. Gina Maria Lucci ${ }^{2}$. Jo-Anne Cavanagh ${ }^{3}$}

Published online: 7 September 2021

(C) Springer-Verlag GmbH Germany, part of Springer Nature 2021

\section{Correction to: Environmental Science and Pollution Research} https://doi.org/10.1007/s11356-021-15513-x

The published paper contains a mistake in Equation 1.

$$
\begin{gathered}
\log [\mathrm{Cd}]_{\text {soil soln }}=6.246( \pm 0.487)-0.987( \pm 0.070) \mathrm{pH}-0.513( \pm 0.128) \log \mathrm{OM}+0.818( \pm 0.133) \log [\mathrm{Cd}]_{\text {total soil }} \\
R^{2} \mathrm{adj}=0.84( \pm 0.17)
\end{gathered}
$$

The original article has been corrected.

Publisher's note Springer Nature remains neutral with regard to jurisdictional claims in published maps and institutional affiliations.

The online version of the original article can be found at https://doi.org/ 10.1007/s11356-021-15513-x

Colin William Gray

colin.gray@agresearch.co.nz

1 AgResearch, Lincoln Research Centre, Private Bag 4749, Christchurch, New Zealand

2 AgResearch Ruakura Research Centre, Private Bag 3123, Hamilton 3240, New Zealand

3 Manaaki-Whenua Landcare Research, 54 Gerald Street, PO Box 69040, Lincoln 7640, New Zealand 